\title{
Sensitivity to encephalitogenic protein in optic neuritis
}

\author{
S. LESSELL, P. BEHAN, J. LAMARGHE, AND M. KIES
}

From the Departments of Ophthalmology and Neurology, Boston University School of Medicine, and the Section on Myelin Chemistry, National Institute of Mental Health, Bethesda, Maryland

Lymphocytes from humans and animals with delayed hypersensitivity undergo morphological changes when cultured with the antigen to which they are sensitive (Robbins, I964). This phenomenon-lymphoblastic transformation-occurs in 5 to 30 per cent. of the sensitized lymphocytes in the presence of the specific antigen and is generally considered a specific indication of delayed hypersensitivity (Oppenheim, r968). Leucocytes from the peripheral blood of patients previously sensitized to tuberculin (by inoculation or infection) transform if cultured in a medium containing tuberculin (Pearmain, Lycette, and Fitzgerald, r 963 ).

Positive correlation with skin tests has been shown in a variety of disorders (Elves, Roath, and Israëls, I963).

Transformation has been demonstrated in a variety of human neurological disorders (including multiple sclerosis) by culturing patients' peripheral lymphocytes in a medium with rabies vaccine of rabbit brain origin (Hashem and Barr, 1963). The antigen responsible for the mitogenic response presumably resides in the brain tissue. In the cells of patients with acute haemorrhagic leucoencephalitis and postinfectious encephalomyelitis, increased lymphoblastic transformation has been demonstrated to a central nervous system myelin basic protein (Behan, Geschwind, Lamarche, Lisak, and Kies, 1968). This protein induces allergic encephalomyelitis when mixed with complete Freund's adjuvant and injected into animals (Kies, 1965). This communication presents evidence of sensitivity to this protein in patients with optic neuritis.

\section{Patients and methods}

\section{A. PATIENTS}

Subjects were culled from a series of 88 patients referred for a study of the natural history of optic neuropathies. Study cases, selected at random, included fourteen individuals with unexplained optic neuritis, seven with optic neuritis associated with multiple sclerosis, and sixteen patients without optic neuritis. All had been diagnosed before the present investigation. The evaluation included complete ophthalmological and neurological histories and physical examinations, skull and orbital $x$ rays, electroencephalogram, brain scan, complete blood count, urine analysis, serological tests for syphilis (Hinton), blood sugar, protein-bound iodine, serum vitamin $B_{12}$ level, and urinary porphyrins and heavy metals. When clinical circumstances dictated, lumbar puncture, pneumoencephalography, carotid angiography, and fluorescein fundus angiography were also performed.

Patients were designated as cases of optic neuritis when there had been a rapid onset of visual impairment with a central, paracentral, or arcuate scotoma, impaired colour perception, and either a normal fundus or disc swelling. There were eleven unilateral and three sequentially involved bilateral uncomplicated cases. None of these had any evidence, by history, examination, or subsequent course, of involvement of the central nervous system. 
The diagnosis of optic neuritis associated with multiple sclerosis was reserved for patients who presented evidence of white matter lesions in addition to the optic neuritis.

Control patients included five normal subjects, three with schizophrenia, two with Leber's heredit-ô ary optic atrophy, three with Alzheimer's disease, one with drusen of the optic nerve, one with choroidal vasculopathy, and one ischaemic optic neuropathy.

\section{B. IMMUNOLOGICAL METHODS}

The antigen used was human encephalitogenic myelin basic protein as prepared by Kies (Kies, $\stackrel{\mathbb{\Omega}}{\mathscr{\Omega}}$ Thompson, and Alvord, 1965). The protein was used at a concentration of 10 gamma $/ \mathrm{ml}$. culture medium unless otherwise stated. Control antigens consisted of purified protein derivative (PPD) $\vec{\odot}$ calf thymus histone, and bovine gamma globulin at the same concentration. Tissue culture fluid $\overrightarrow{ }$ consisted of a stock solution of TC 19 (Microbiological Associates) $500 \mathrm{ml}$., penicillin/streptomycin ${ }_{\odot}^{\omega}$ (Microbiological Associates) 5,000 units/ml.5 ml. and heparin (Abbott) ro,ooo units $5 \mathrm{ml}$. TheO culture flasks were sterilized screw-top vials $(20 \mathrm{ml}$.) as used in liquid scintillation counting (Nuclear: Chicago).

60 to $80 \mathrm{ml}$. of venous blood were withdrawn from each subject into a heparinized plastic syringe.. The syringe was left standing upright for io to 30 minutes to permit sedimentation of red blood cells. The supernatant, containing the lymphocytes, was removed and centrifuged at 700 RPM for 5 min-o utes. The pellet was re-suspended in tissue culture fluid and washed twice by repeated centrifugation. After the final washing, the cell concentration was adjusted to give $1-2 \times 10^{6} \mathrm{cells} / \mathrm{ml} . Z$ tissue culture fluid. Individual cultures consisted of $\mathrm{I} \mathrm{ml}$. per flask. 150 to 200 cultures were run for each patient as follows.:

$\begin{array}{ll}\text { Human encephalitogenic basic protein } & 30 \\ \text { PPD } & 30 \\ \text { Bovine gamma globulin } & 30 \\ \text { Calf thymus histone } & 30 \\ \text { Control (nothing added) } & 30 \\ \text { Phytohaemagglutinin (PHA) } & \text { 10 }\end{array}$

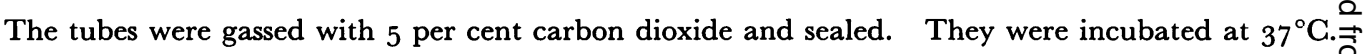
for 6 days, except for the PAH cultures which were harvested at 3 days. All cultures had a 24-hour 3 pulse before harvest of $2.5 \mathrm{mc}$. tritiated thymidine, specific activity $6.7 \mathrm{c} / \mathrm{mM}$. (New England Nuclear).

After harvesting, the cells were washed twice in normal saline and the cellular pellet was frozeno. overnight. They were allowed to that at room temperature. I ml. cold ro per cent. trichloracetic:acid was added to each tube and the cells were re-suspended. They were then spun at 1,500 r.p.m. $\cong$ for 10 minutes. The trichloracetic acid was decanted and the button allowed to drain upright for 3 minutes to permit drainage of the supernatant fluid. The trichloracetic acid treatment was repeated three times. The cellular pellet was then dissolved in $0.5 \mathrm{ml}$. NCS (Nuclear Chicago)윽 reagent. This was usually accomplished by gentle intermittent agitation of the solution for 5 hours at room temperature. After the cells were completely dissolved, io ml. Bray's solution were addedㅡㅡㄹ and the clear solution was put in scintillation vials. All centrifugation and trichloracetic acidN precipitations were done at $4^{\circ} \mathrm{C}$.

Each vial was counted for 30 minutes and the counts/minute were determined after correction for $\mathcal{N}^{\circ}$ efficiency and quenching. The mean of each set of cultures was noted and the results expressed as $\mathrm{G}$ the ratio:

counts/minute with antigen

counts/minute without antigen (control)

A small sample of cells was removed from I-2 culture flasks of each set for autoradiography. 


\section{Results}

The results for the myelin protein, PPD, and bovine gamma globulin are presented in Figures I, 2, and 3 respectively. There was a good correlation between the scintillation counting results and the radioautographs.

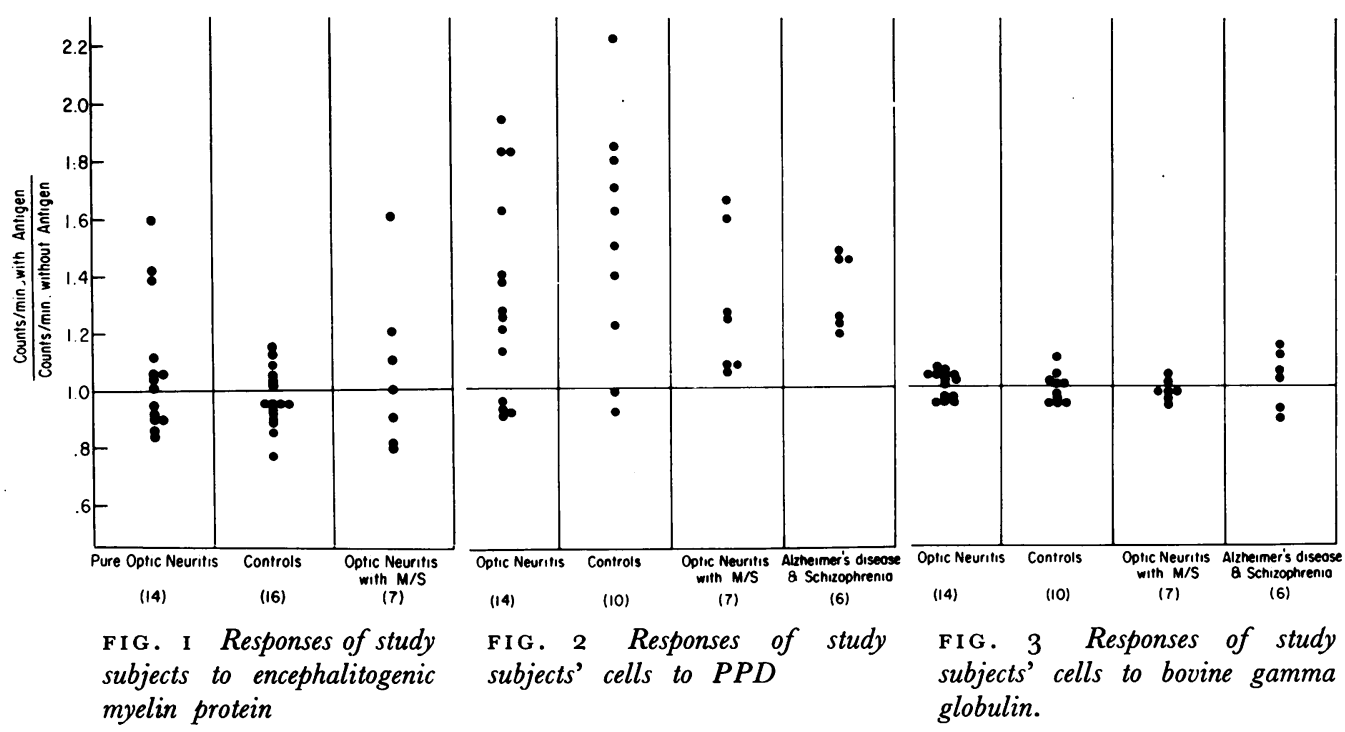

Inspection of the results with the myelin protein suggests that a ratio of $\mathrm{I}: 2 \mathrm{might}$ be arbitrarily employed to divide the subjects into those who did and those who did not transform. On that basis three uncomplicated optic neuritis patients and one with optic neuritis associated with multiple sclerosis would be considered "positive".

Their clinical histories are summarized as follows:

Case I (Optic neuritis associated with multiple sclerosis)

A 32-year-old man awoke one morning with pain and blurred vision in the left eye. Colours appeared "washed out" with that eye. Examination revealed normal vision in the right eye. The left eye had a visual acuity of counting fingers at 4 feet, a dense central scotoma, and impaired colour perception. The left disc was oedematous.

Complete evaluation was negative and vision improved to 20/20 without medication, but 6 weeks later he developed sensory evidence of a myelopathy at the $D_{3}$ level. This later cleared, but 6 months later he developed evanescent hyperaesthesia and dysaesthesia of the left hand.

Case 2 (Uncomplicated optic neuritis)

A 23-year-old woman noted pain on moving the right eye; 2 days later the vision became "foggy" in that eye. The left eye had a relative paracentral scotoma and impaired colour sense. Both fundi were normal.

Complete evaluation was negative and 6 months later this woman's right eye had 20/1 3 vision, full field, and unimpaired colour perception.

Case 3 (Uncomplicated optic neuritis)

A 4 I-year-old woman suddenly developed impaired vision in the right eye unaccompanied by pain. The vision was found to be $20 / 200$ in the right eye and $20 / 20$ in the left. There was a central scotoma and impaired colour perception. The fundi were unremarkable. 
Complete evaluation was negative. Without medication the vision returned to 20/25 although pathological temporal pallor became evident in the right eye.

Case 4 (Uncomplicated optic neuritis)

A 39-year-old woman awoke with blurred vision and pain in the right eye. Vision was found to be $\stackrel{\overrightarrow{5}}{\rightarrow}$ $20 / 25$ in the right eye and $20 / 20$ in the left. The right eye had impaired colour perception and an

arcuate scotoma. The fundi were normal.
Complete evaluation was negative. The vision returned to $20 / 20$ and colour perception became normal without medication, but a small relative paracentral scotoma remained.

The mean ratio of response to the encephalitogenic protein for the entire population was $\overrightarrow{0}$ I 05. The standard deviation was 6.2. Only two patients (Case I, with multiple $\overrightarrow{ }$ sclerosis, and Case 2, with uncomplicated optic neuritis-above) fell more than two standard deviations above the mean. Transformation to the encephalitogenic protein $\frac{0}{0}$. was not encountered among controls.

Gamma globulin failed to induce transformation in the lymphocytes of any of the subjects. Transformation was equally prevalent in all groups of study subjects when PPD was used.

\section{Discussion}

Optic neuritis may herald, accompany, or follow the brain and cord lesions of multiple $\vec{z}$ sclerosis. II 5 to 85 per cent. of patients with uncomplicated first attacks of optic neuritis are later found to have evidence of disseminated central nervous system demyelination (Kurland, Auth, Beebe, Kurtze, Lessell, Nagler, and Nefzger, r963; McAlpine, r96r). Attempts to identify features that would indicate which patients with uncomplicated opti $\vec{\varphi}$ neuritis will develop additional white-matter lesions have failed (Bradley and Whitty, 1968.

The present study supports, on immunological grounds, a relationship between u complicated optic neuritis and other white-matter diseases of the central nervous system that has been previously established clinically, pathologically, and epidemiologically. Should autosensitivity be responsible for spontaneous demyelinating disorders one would expect the antigen to reside in myelin. This is the case in experimental allergic encephalo- $\stackrel{\mathbb{Q}}{\triangle}$ myelitis. The positive lymphoblastic transformation results in cases of acute haemorrhagic $\overline{\vec{a}}$ leucoencephalitis and postinfectious encephalomyelitis suggest that these disorders in man are the counterpart of experimental allergic encephalomyelitis in the animal. The antigen used in the present study was a human myelin component that is capable of inducing allergic encephalitis in susceptible animals with as little as 5 gamma. While only two of our cases showed sensitivity to this component, this may be explained by the 3 . relationship of the test to the time of onset of the disease. Fig. 4 shows the relationship 8 of response to the time at which the test was done in patients with uncomplicated optic 3 neuritis. In a separate study it was found that lymphoblastic transformation, when $\frac{9}{9}$ performed serially in monkeys with experimental allergic encephalomyelitis, showed a time variation (Kies and others, 1965). Since animals with experimental allergic encephalomyelitis show decreased hypersensitivity after the onset of disease (Shaw, Alvord, Kaku, $\stackrel{N}{\mathscr{O}}$ and Kies, I965), the immunological responses may vary with time in other demyelinating $\mathbb{O}^{\circ}$ diseases. Fig. 5 shows that a time correlation could not be found in patients with optic $\mathbb{E}^{N}$ neuritis associated with multiple sclerosis. Other possible explanations for our failure to find a higher percentage of positives include the possibilities that more than one antigen is responsible for the disease, that optic neuritis is an expression of many different pathological conditions, and that hypersensitivity may be the result rather than the cause of the disease. 

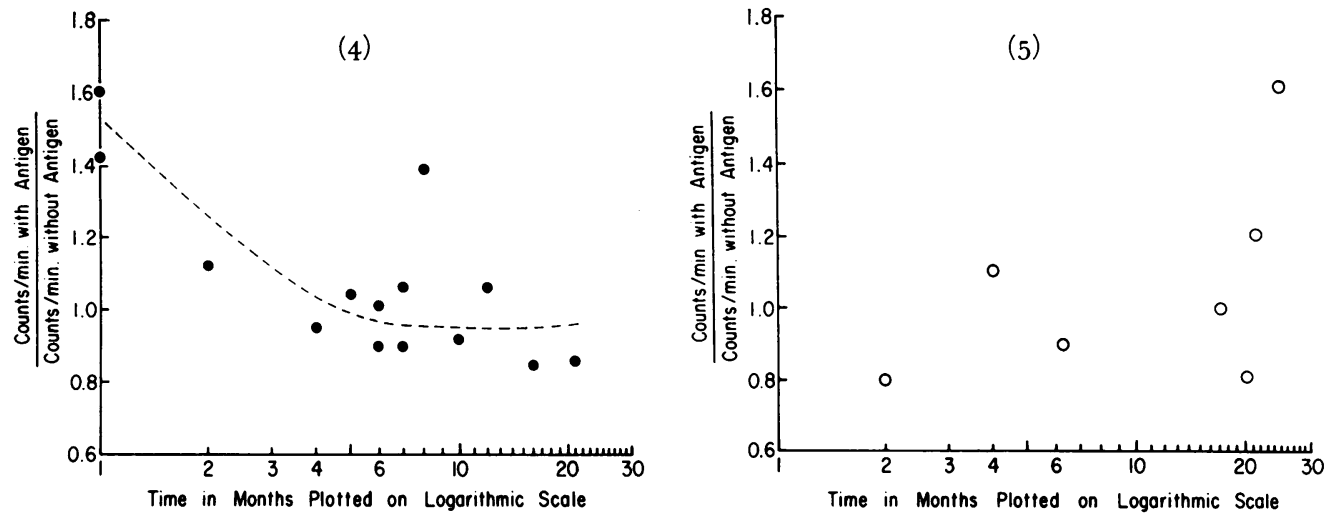

FIG. 4 Relationship between cell response and interval since onset of uncomplicated optic neuritis

FIG. 5 Relationship between cell response and interval since onset of optic neuritis with multiple sclerosis

Recently several observers have noted the occurrence of lymphocyte transformation in sensitized experimental animals which did not demonstrate delayed hypersensitivity. Benezra, Gery, and Davies ( 1969) demonstrated transformation in rabbits who had marked Arthus reactions but no skin responses of the delayed hypersensitivity type. Schlossman, Herman, and Yarron ( 1969 ) found poor correlation between skin tests and transformation. These observations would suggest that transformation could indicate either a humorally or cellularly mediated response. On the other hand, Cooperband, Rosen, and Kibrick (1968) showed that lymphocyte transformation proceeds in a normal way in humans with acquired, X-linked, and the sporadic congenital varieties of agammaglobulinaemia. This would tend to suggest that humoral factors do not play a role.

\section{Summary}

Lymphoblastic transformation to a human myelin encephalitogenic protein was evaluated in fourteen cases of uncomplicated optic neuritis, seven cases of optic neuritis associated with multiple sclerosis, and sixteen controls. Transformation was evident in one patient with uncomplicated optic neuritis and one patient with optic neuritis associated with multiple sclerosis. These studies support, on an immunological basis, a relationship between optic neuritis and other white-matter disorders of the central nervous system.

\section{References}

Behan, P. O., GeSchWind, N., LAMARGhe, J. B., LiSAK, R. P., and KiES, M. W. (I968) Lancet, 2, IOo9 Benezra, D., GERY, I., and DAVIES, A. M. (1969) Clin. exp. Immunol., 5, I 55

Bradley, w. G., and whitty, c. W. m. (1968) J. Neurol. Neurosurg. Psychiat., 31, ro

COOPERBAND, s. R., ROSEN, F. s., and KIBRICK, s. (ig68) $\quad$ J. clin. Invest., 47, 836

ELVES, M. W., ROATH, S., and ISRAËLS, M. G.,(1963) Lancet, r, 806

HASHEM, N., and BARR, M. L. (1963) Ibid., 2, IO29

кIES, м. W. (1965) Ann. N.Y. Acad. Sci., r22, r6 I

THOMPSON, E. B., and ALVORD, E. c. (1965) Ibid., 122, 148

KURland, L. T., AUTh, T. H., BeEbe, G. W., KURTZE, J. F., LeSSEll, S., NAGLer, B., and NEFZGER, M. D.

(1963) Trans. Amer. neurol. Ass., 88, $23 \mathrm{I}$

MGALPINE, D. ( I96I) Brain, 84, 186

oppenheim, J. J. (I968) Fed. Proc., 27, 2 I

PEARMAin, G., LYGeTte, R. R., and FITZGerald, P. H. (1963) Lancet, r, 637

RoBbins, J. H. (1964) Science, 146, 1648

schlossman, s. F., Herman, J., and yaron, A. (1969) J. exp. Med., r30, 103 I

Shaw, Gheng-mei, Alvord, E. G., KAKU, J., and kies, m. w. (I965) Ann. N.Y. Acad. Sci., I22, 3 I 8 